\title{
The smallest one-realization of a given set
}

\author{
Ping Zhao \\ School of Science, Linyi University, \\ Linyi, Shandong, 276005, China
}

zhaopingly@163.com

\author{
Kefeng Diao \\ School of Science, Linyi University, \\ Linyi, Shandong, 276005, China \\ kfdiao@163.com
}

\author{
Kaishun Wang* \\ Sch. Math. Sci. \& Lab. Math. Com. Sys. \\ Beijing Normal University, Beijing 100875, China \\ wangks@bnu.edu.cn
}

Submitted: Oct 26, 2011; Accepted: Dec 9, 2011; Published: Jan 16, 2012

Mathematics Subject Classifications: 05C15, 05C65, 05C85

\begin{abstract}
For any set $S$ of positive integers, a mixed hypergraph $\mathcal{H}$ is a realization of $S$ if its feasible set is $S$, furthermore, $\mathcal{H}$ is a one-realization of $S$ if it is a realization of $S$ and each entry of its chromatic spectrum is either 0 or 1 . Jiang et al. showed that the minimum number of vertices of a realization of $\{s, t\}$ with $2 \leq s \leq t-2$ is $2 t-s$. Král proved that there exists a one-realization of $S$ with at most $|S|+2 \max S-\min S$ vertices. In this paper, we determine the number of vertices of the smallest onerealization of a given set. As a result, we partially solve an open problem proposed by Jiang et al. in 2002 and by Král in 2004.
\end{abstract}

Key words: hypergraph coloring; mixed hypergraph; feasible set; chromatic spectrum; one-realization

\section{Introduction}

A mixed hypergraph on a finite set $X$ is a triple $\mathcal{H}=(X, \mathcal{C}, \mathcal{D})$, where $\mathcal{C}$ and $\mathcal{D}$ are families of subsets of $X$, called the $\mathcal{C}$-edges and $\mathcal{D}$-edges, respectively. A bi-hypergraph is a mixed hypergraph with $\mathcal{C}=\mathcal{D}$. A sub-hypergraph $\mathcal{H}^{\prime}=\left(X^{\prime}, \mathcal{C}^{\prime}, \mathcal{D}^{\prime}\right)$ of a mixed hypergraph $\mathcal{H}=(X, \mathcal{C}, \mathcal{D})$ is a spanning sub-hypergraph if $X^{\prime}=X$, and $\mathcal{H}^{\prime}$ is called a derived sub-hypergraph of $\mathcal{H}$ on $X^{\prime}$, denoted by $\mathcal{H}\left[X^{\prime}\right]$, when $\mathcal{C}^{\prime}=\left\{C \in \mathcal{C} \mid C \subseteq X^{\prime}\right\}$ and $\mathcal{D}^{\prime}=\left\{D \in \mathcal{D} \mid D \subseteq X^{\prime}\right\}$. Two mixed hypergraphs $\mathcal{H}_{1}=\left(X_{1}, \mathcal{C}_{1}, \mathcal{D}_{1}\right)$ and $\mathcal{H}_{2}=\left(X_{2}, \mathcal{C}_{2}, \mathcal{D}_{2}\right)$

\footnotetext{
*Corresponding author
} 
are isomorphic if there exists a bijection $\phi$ from $X_{1}$ to $X_{2}$ that preserves the incidence between vertices and edges and maps each $\mathcal{C}$-edge of $\mathcal{C}_{1}$ onto a $\mathcal{C}$-edge of $\mathcal{C}_{2}$ and maps each $\mathcal{D}$-edge of $\mathcal{D}_{1}$ onto a $\mathcal{D}$-edge of $\mathcal{D}_{2}$, and vice versa. The bijection $\phi$ is called an isomorphism from $\mathcal{H}_{1}$ to $\mathcal{H}_{2}$.

A proper $k$-coloring of $\mathcal{H}$ is a mapping from $X$ into a set of $k$ colors so that each $\mathcal{C}$-edge has two vertices with a Common color and each $\mathcal{D}$-edge has two vertices with Distinct colors. A strict $k$-coloring is a proper $k$-coloring using all of the $k$ colors, and a mixed hypergraph is $k$-colorable if it has a strict $k$-coloring. The maximum (minimum) number of colors in a strict coloring of $\mathcal{H}=(X, \mathcal{C}, \mathcal{D})$ is the upper chromatic number $\bar{\chi}(\mathcal{H})$ (resp. lower chromatic number $\chi(\mathcal{H})$ ) of $\mathcal{H}$. The study of the colorings of mixed hypergraphs has made a lot of progress since its inception [7]. For more information, we would like refer readers to $[3,6,8,9]$.

A coloring of $\mathcal{H}$ may be viewed as a partition of the vertex set, where each color class consists of vertices assigned to the same color. Then no class contains a $\mathcal{D}$-edge, and each $\mathcal{C}$-edge meets some class in more than one vertex. Such partitions are called feasible partitions. So a strict $n$-coloring $c=\left\{C_{1}, C_{2}, \ldots, C_{n}\right\}$ of a mixed hypergraph means that $C_{1}, C_{2}, \ldots, C_{n}$ are the $n$ color classes under $c$.

The set of all the values $k$ such that $\mathcal{H}$ has a strict $k$-coloring is called the feasible set of $\mathcal{H}$, denoted by $\mathcal{F}(\mathcal{H})$. For each $k$, let $r_{k}$ denote the number of partitions of the vertex set corresponding to the strict colorings of $\mathcal{H}$ with $k$ colors. The vector $R(\mathcal{H})=\left(r_{1}, r_{2}, \ldots, r_{\bar{\chi}}\right)$ is called the chromatic spectrum of $\mathcal{H}$. A mixed hypergraph has a gap at $k$ if its feasible set contains elements larger and smaller than $k$ but omits $k$. A gap of size $g$ means $g$ consecutive gaps. If some gaps occur, the feasible set and the chromatic spectrum of $\mathcal{H}$ are said to be broken, and if there are no gaps then they are called continuous or gap-free. If $S$ is a set of positive integers, we say that a mixed hypergraph $\mathcal{H}$ is a realization of $S$ if $\mathcal{F}(\mathcal{H})=S$. A mixed hypergraph $\mathcal{H}$ is a one-realization of $S$ if it is a realization of $S$ and all the entries of the chromatic spectrum of $\mathcal{H}$ are either 0 or 1 . This concept was firstly introduced by Král [4].

Bujtás et al. [1] gave a necessary and sufficient condition for a set $S$ to be the feasible set of an $r$-uniform mixed hypergraph. Kündgen et al. [5] found a one-realization of $\{2,4\}$ on 6 vertices for planar hypergraphs. Jiang et al. [2] proved that a set $S$ of positive integers is a feasible set of a mixed hypergraph if and only if $1 \notin S$ or $S$ is an interval. They also discussed the bound on the number of vertices of a mixed hypergraph with a gap, in particular, the minimum number of vertices of a realization of $\{s, t\}$ with $2 \leq s \leq t-2$ is $2 t-s$. Moreover, they also mentioned that the question of finding the minimum number of vertices in a mixed hypergraph with feasible set $S$ of size at least 3 remains open. In [10], we obtained an upper bound on the minimum number of vertices of 3-uniform bihypergraphs with a given feasible set. Král [4] proved that there exists a one-realization of $S$ with at most $|S|+2 \max S-\min S$ vertices, and proposed the following problem: what is the number of vertices of the smallest mixed hypergraph whose spectrum is equal to a given spectrum $\left(r_{1}, r_{2}, \ldots, r_{m}\right)$ ?

In this paper, we determine the number of vertices of the smallest one-realization of a given set and obtain the following result: 
Theorem 1.1 For any integers $2 \leq n_{s}<\cdots<n_{2}<n_{1}$, let $\delta(S)$ denote the number of vertices of the smallest one-realization of $S=\left\{n_{1}, n_{2}, \ldots, n_{s}\right\}$. Then

$$
\delta(S)= \begin{cases}2 n_{1}-n_{s}, & \text { if } n_{1}>n_{2}+1 \\ 2 n_{1}-n_{s}-1, & \text { if } n_{1}=n_{2}+1\end{cases}
$$

As a result, we partially solve the above open problem proposed by Jiang et al. and by Král.

\section{Proof of Theorem 1.1}

In this section we always assume that $S=\left\{n_{1}, n_{2}, \ldots, n_{s}\right\}$ is a set of integers with $2 \leq n_{s}<\cdots<n_{2}<n_{1}$. We first show that the number $\delta(S)$ given in Theorem 1.1 is a lower bound on the number of vertices of the smallest one-realization of $S$, then construct two families of mixed hypergraphs which meet the bounds.

Jiang et al. [2] discussed the bound on the number of vertices of a mixed hypergraph with a gap.

Proposition 2.1 ([2, Theorem 3]) If $\mathcal{H}=(X, \mathcal{C}, \mathcal{D})$ is an s-colorable mixed hypergraph with a gap at $t-1$, then $|X| \geq 2 t-s$. For $2 \leq s \leq t-2$, this bound is sharp.

Lemma 2.2

$$
\delta(S) \geq \begin{cases}2 n_{1}-n_{s}, & \text { if } n_{1}>n_{2}+1 \\ 2 n_{1}-n_{s}-1, & \text { if } n_{1}=n_{2}+1\end{cases}
$$

Proof. Assume that $\mathcal{H}=(X, \mathcal{C}, \mathcal{D})$ is a one-realization of $S$.

Case 1. $n_{1}>n_{2}+1$. Then $\mathcal{H}$ has a gap at $n_{1}-1$. By Proposition 2.1, we have $\delta(S) \geq 2 n_{1}-n_{s}$.

Case 2. $n_{1}=n_{2}+1$. Suppose $|X| \leq 2 n_{1}-\left(n_{s}+2\right)$. For any strict $n_{1}$-coloring $c_{1}=\left\{C_{1}, C_{2}, \ldots, C_{n_{1}}\right\}$ of $\mathcal{H}$, there exist at least $n_{s}+2$ color classes of size one. Suppose $C_{1}=\left\{\alpha_{1}\right\}, C_{2}=\left\{\alpha_{2}\right\}, \ldots, C_{n_{s}+2}=\left\{\alpha_{n_{s}+2}\right\}$. For any strict $n_{s}$-coloring $c_{s}$ of $\mathcal{H}$, there are the following two possible cases.

Case 2.1. There exist three vertices in $\left\{\alpha_{1}, \alpha_{2}, \ldots, \alpha_{n_{s}+2}\right\}$ which fall into a common color class under $c_{s}$. Suppose $\alpha_{1}, \alpha_{2}, \alpha_{3}$ are in a common color class under $c_{s}$. Then $\left\{\alpha_{1}, \alpha_{2}\right\},\left\{\alpha_{1}, \alpha_{3}\right\},\left\{\alpha_{2}, \alpha_{3}\right\} \notin \mathcal{D}$, which implies that $\left\{C_{1} \cup C_{2}, C_{3}, \ldots, C_{n_{1}}\right\},\left\{C_{1} \cup\right.$ $\left.C_{3}, C_{2}, C_{4}, \ldots, C_{n_{1}}\right\},\left\{C_{1}, C_{2} \cup C_{3}, C_{4}, \ldots, C_{n_{1}}\right\}$ are strict $n_{2}$-colorings of $\mathcal{H}$. Therefore, $\mathcal{H}$ is not a one-realization of $S$, a contradiction.

Case 2.2. There exist two pairs of vertices in $\left\{\alpha_{1}, \alpha_{2}, \ldots, \alpha_{n_{s}+2}\right\}$ each of which falls into a common color class under $c_{s}$. Suppose $\alpha_{1}, \alpha_{2}$ are in a common color class and $\alpha_{3}, \alpha_{4}$ are in common color class under $c_{s}$. Then $\left\{\alpha_{1}, \alpha_{2}\right\},\left\{\alpha_{3}, \alpha_{4}\right\} \notin \mathcal{D}$. It follows that $\left\{C_{1} \cup C_{2}, C_{3}, \ldots, C_{n_{1}}\right\}$ and $\left\{C_{1}, C_{2}, C_{3} \cup C_{4}, C_{5}, \ldots, C_{n_{1}}\right\}$ are strict $n_{2}$-colorings of $\mathcal{H}$. Then $\mathcal{H}$ is not a one-realization of $S$, a contradiction. Hence, $\delta(S) \geq 2 n_{1}-n_{s}-1$. 
In the rest of this section, we shall construct two families of mixed hypergraphs which meet the bound in Lemma 2.2.

For any positive integer $n$, let $[n]$ denote the set $\{1,2, \ldots, n\}$.

Construction I. For any positive integer $s \geq 2$, let

$$
\begin{aligned}
& X_{n_{1}, \ldots, n_{s}}^{0}=\left\{(\underbrace{i, i, \ldots, i}_{s}) \mid i=1,2, \ldots, n_{s}-1\right\}, \\
& X_{n_{1}, \ldots, n_{s}}^{1}=\bigcup_{t=2}^{s} \bigcup_{j=n_{t}}^{n_{t-1}-1}\left\{(\underbrace{j, \ldots, j}_{t-1}, n_{t}, n_{t+1}, \ldots, n_{s}),(\underbrace{j, \ldots, j}_{t-1}, \underbrace{1, \ldots, 1}_{s-t+1})\right\} .
\end{aligned}
$$

Suppose

$$
\begin{aligned}
& X_{n_{1}, \ldots, n_{s}}^{*}=X_{n_{1}, \ldots, n_{s}}^{0} \cup X_{n_{1}, \ldots, n_{s}}^{1} \cup\left\{\left(n_{1}, n_{2}, \ldots, n_{s}\right)\right\} \\
& \mathcal{D}_{n_{1}, \ldots, n_{s}}^{*}=\left\{\left\{\left(x_{1}, x_{2}, \ldots, x_{s}\right),\left(y_{1}, y_{2}, \ldots, y_{s}\right)\right\} \mid x_{i} \neq y_{i}, i \in[s]\right\}, \\
& \mathcal{C}_{n_{1}, \ldots, n_{s}}^{*}=\left\{\left\{\left(x_{1}, \ldots, x_{s}\right),\left(y_{1}, \ldots, y_{s}\right),\left(z_{1}, \ldots, z_{s}\right)\right\}||\left\{x_{j}, y_{j}, z_{j}\right\} \mid=2, j \in[s]\right\} .
\end{aligned}
$$

Then $\mathcal{H}_{n_{1}, \ldots, n_{s}}^{*}=\left(X_{n_{1}, \ldots, n_{s}}^{*}, \mathcal{C}_{n_{1}, \ldots, n_{s}}^{*}, \mathcal{D}_{n_{1}, \ldots, n_{s}}^{*}\right)$ is a mixed hypergraph with $2 n_{1}-n_{s}$ vertices.

Let

$$
\begin{gathered}
X_{n_{1}, \ldots, n_{s}}=\left\{\left(x_{1}, x_{2}, \ldots, x_{s}\right) \mid x_{i} \in\left[n_{i}\right], i \in[s]\right\}, \\
X_{i j}^{s}=\left\{\left(x_{1}, x_{2}, \ldots, x_{i-1}, j, x_{i+1}, \ldots, x_{s}\right) \mid x_{k} \in\left[n_{k}\right], k \in[s] \backslash\{i\}\right\}, j \in\left[n_{i}\right] .
\end{gathered}
$$

Then, for any $i \in[s]$,

$$
c_{i}^{s *}=\left\{X_{i 1}^{*}, X_{i 2}^{*}, \ldots, X_{i n_{i}}^{*}\right\}
$$

is a strict $n_{i}$-coloring of $\mathcal{H}_{n_{1}, \ldots, n_{s}}^{*}$, where $X_{i j}^{*}=X_{n_{1}, \ldots, n_{s}}^{*} \cap X_{i j}^{s}, j \in\left[n_{i}\right]$.

For the case of $s=3, n_{1}=7, n_{2}=4, n_{3}=2$, we have

$$
\begin{aligned}
X_{7,4,2}^{*} & =\{(1,1,1)\} \cup\{(2,2,2),(2,2,1),(3,3,2),(3,3,1)\} \\
& \cup\{(4,4,2),(4,1,1),(5,4,2),(5,1,1),(6,4,2),(6,1,1)\} \cup\{(7,4,2)\} .
\end{aligned}
$$

Lemma $2.3 \mathcal{H}_{n_{1}, n_{2}}^{*}$ is a one-realization of $\left\{n_{1}, n_{2}\right\}$.

Proof. Under any strict coloring $c=\left\{C_{1}, C_{2}, \ldots, C_{m}\right\}$ of $\mathcal{H}_{n_{1}, n_{2}}^{*}$, the vertices $(1,1)$, $(2,2), \ldots,\left(n_{2}, n_{2}\right)$ fall into distinct color classes. For each $i \in\left[n_{2}\right]$, suppose $(i, i) \in C_{i}$. Then, for any $i \in\left[n_{2}-1\right]$ and $j \in\left[n_{1}-n_{2}-1\right]$, the $\mathcal{D}$-edge $\left\{\left(n_{2}+j, n_{2}\right),(i, i)\right\}$ implies that $\left(n_{2}+j, n_{2}\right) \notin C_{i}$ and the $\mathcal{D}$-edge $\left\{\left(n_{2}+j, 1\right),\left(n_{2}, n_{2}\right)\right\}$ implies that $\left(n_{2}+j, 1\right) \notin C_{n_{2}}$. Since $\left\{(1,1),\left(n_{2}, 1\right),\left(n_{2}, n_{2}\right)\right\}$ is a $\mathcal{C}$-edge, $\left(n_{2}, 1\right) \in C_{1} \cup C_{n_{2}}$.

Case 1. $\left(n_{2}, 1\right) \in C_{1}$. The fact that $\left\{\left(n_{2}, 1\right),\left(n_{2}, n_{2}\right),\left(n_{2}+1, n_{2}\right)\right\}$ is a $\mathcal{C}$-edge follows that $\left(n_{2}+1, n_{2}\right) \in C_{n_{2}}$. From the $\mathcal{C}$-edge $\left\{\left(n_{2}, 1\right),\left(n_{2}+1,1\right),\left(n_{2}+1, n_{2}\right)\right\}$, we observe $\left(n_{2}+1,1\right) \in C_{1}$. Similarly, $\left(n_{2}+j, 1\right) \in C_{1},\left(n_{2}+j, n_{2}\right) \in C_{n_{2}}$ for any $j \in\left[n_{1}-n_{2}-1\right]$ and $\left(n_{1}, n_{2}\right) \in C_{n_{2}}$. Therefore, $c=c_{2}^{2 *}$.

Case 2. $\left(n_{2}, 1\right) \in C_{n_{2}}$. The $\mathcal{D}$-edge $\left\{\left(n_{2}, 1\right),\left(n_{2}+1, n_{2}\right)\right\}$ implies that $\left(n_{2}+1, n_{2}\right) \notin C_{n_{2}}$. Suppose $\left(n_{2}+1, n_{2}\right) \in C_{n_{2}+1}$. From the $\mathcal{C}$-edge $\left\{\left(n_{2}, 1\right),\left(n_{2}+1,1\right),\left(n_{2}+1, n_{2}\right)\right\}$, we have 
$\left(n_{2}+1,1\right) \in C_{n_{2}+1}$. Similarly, $\left(n_{2}+j, n_{2}\right),\left(n_{2}+j, 1\right) \in C_{n_{2}+j}$ for any $j \in\left[n_{1}-n_{2}-1\right]$ and $\left(n_{1}, n_{2}\right) \in C_{n_{1}}$. Therefore, $c=c_{1}^{2 *}$.

Hence, the desired result follows.

Theorem 2.4 $\mathcal{H}_{n_{1}, \ldots, n_{s}}^{*}$ is a one-realization of $S$.

Proof. By Lemma 2.3, the conclusion is true for $s=2$.

Let $X^{\prime}=\left\{\left(x_{2}, x_{2}, x_{3}, \ldots, x_{s}\right) \mid x_{j} \in\left[n_{j}\right], j \in[s] \backslash\{1\}\right\}$. Then $\mathcal{H}^{\prime}=\mathcal{H}_{n_{1}, \ldots, n_{s}}^{*}\left[X^{\prime}\right]$ is isomorphic to $\mathcal{H}_{n_{2}, n_{3}, n_{4}, \ldots, n_{s}}^{*}$. By induction, all the strict colorings of $\mathcal{H}^{\prime}$ are as follows:

$$
c_{i}^{\prime}=\left\{X_{i 1}^{\prime}, X_{i 2}^{\prime}, \ldots, X_{i n_{i}}^{\prime}\right\}, \quad i \in[s] \backslash\{1\},
$$

where $X_{i j}^{\prime}=X^{\prime} \cap X_{i j}^{*}, j \in\left[n_{i}\right]$. For any strict coloring $c=\left\{C_{1}, \ldots, C_{m}\right\}$ of $\mathcal{H}_{n_{1}, \ldots, n_{s}}^{*}$, the vertices $(1,1, \ldots, 1),(2,2, \ldots, 2), \ldots,\left(n_{s}, n_{s}, \ldots, n_{s}\right)$ fall into distinct color classes. Without loss of generality, suppose $(i, i, \ldots, i) \in C_{i}$ for any $i \in\left[n_{s}\right]$. Then there are the following two possible cases.

Case 1. $\left.c\right|_{X^{\prime}}=c_{2}^{\prime}$. The $\mathcal{C}$-edge $\left\{(1,1, \ldots, 1),\left(n_{2}, 1, \ldots, 1\right),\left(n_{2}, n_{2}, n_{3}, \ldots, n_{s}\right)\right\}$ implies that $\left(n_{2}, 1, \ldots, 1\right) \in C_{1} \cup C_{n_{2}}$.

Case 1.1. $\left(n_{2}, 1, \ldots, 1\right) \in C_{1}$. From the $\mathcal{D}$-edge $\left\{(1, \ldots, 1),\left(n_{2}+1, n_{2}, n_{3}, \ldots, n_{s}\right)\right\}$ and the $\mathcal{C}$-edge $\left\{\left(n_{2}, n_{2}, n_{3}, \ldots, n_{s}\right),\left(n_{2}+1, n_{2}, n_{3}, \ldots, n_{s}\right),\left(n_{2}, 1, \ldots, 1\right)\right\}$, we observe $\left(n_{2}+\right.$ $\left.1, n_{2}, n_{3}, \ldots, n_{s}\right) \in C_{n_{2}}$. By the $\mathcal{C}$-edge $\left\{\left(n_{2}, n_{2}, n_{3}, \ldots, n_{s}\right),\left(n_{2}+1,1, \ldots, 1\right),\left(n_{2}, 1, \ldots, 1\right)\right\}$ and the $\mathcal{D}$-edge $\left\{\left(n_{2}, n_{2}, n_{3}, \ldots, n_{s}\right),\left(n_{2}+1,1, \ldots, 1\right)\right\}$, we observe $\left(n_{2}+1,1, \ldots, 1\right) \in C_{1}$. Similarly, $\left(n_{2}+j, 1, \ldots, 1\right) \in C_{1},\left(n_{2}+j, n_{2}, n_{3}, \ldots, n_{s}\right) \in C_{n_{2}}$ for any $j \in\left[n_{1}-n_{2}-1\right]$ and $\left(n_{1}, n_{2}, \ldots, n_{s}\right) \in C_{n_{2}}$. Therefore, $c=c_{2}^{s *}$.

Case 1.2. $\left(n_{2}, 1, \ldots, 1\right) \in C_{n_{2}}$. Note that $\left(n_{2}+j, 1, \ldots, 1\right) \notin C_{k}$ for any $j \in$ $\left[n_{1}-n_{2}-1\right]$ and $k \in\left[n_{2}\right] \backslash\{1\}$. If $\left(n_{2}+1,1, \ldots, 1\right) \in C_{1}$, from the $\mathcal{C}$-edge $\left\{\left(n_{2}+\right.\right.$ $\left.1,1, \ldots, 1),\left(n_{2}, n_{2}, n_{3}, \ldots, n_{s}\right),\left(n_{2}+1, n_{2}, \ldots, n_{s}\right)\right\}$, we observe $\left(n_{2}+1, n_{2}, \ldots, n_{s}\right) \in C_{1} \cup$ $C_{n_{2}}$, contrary to the fact that both $\left\{(1,1, \ldots, 1),\left(n_{2}+1, n_{2}, \ldots, n_{s}\right)\right\}$ and $\left\{\left(n_{2}, 1, \ldots, 1\right)\right.$, $\left.\left(n_{2}+1, n_{2}, \ldots, n_{s}\right)\right\}$ are $\mathcal{D}$-edges. Then, $\left(n_{2}+1,1, \ldots, 1\right) \notin C_{1}$. Suppose $\left(n_{2}+1,1, \ldots, 1\right) \in$ $C_{n_{2}+1}$. The $\mathcal{C}$-edge $\left\{\left(n_{2}+1,1, \ldots, 1\right),\left(n_{2}+1, n_{2}, n_{3}, \ldots, n_{s}\right),\left(n_{2}, 1, \ldots, 1\right)\right\}$ implies $\left(n_{2}+\right.$ $\left.1, n_{2}, \ldots, n_{s}\right) \in C_{n_{2}+1}$. Similarly, $\left(n_{2}+j, 1, \ldots, 1\right),\left(n_{2}+j, n_{2}, \ldots, n_{s}\right) \in C_{n_{2}+j}$ for any $j \in\left[n_{1}-n_{2}-1\right]$ and $\left(n_{1}, n_{2}, \ldots, n_{s}\right) \in C_{n_{1}}$. Therefore, $c=c_{1}^{s *}$.

Case 2. There exists a $k \in[s] \backslash\{1,2\}$ such that $\left.c\right|_{X^{\prime}}=c_{k}^{\prime}$. In this case, we have $\left(n_{2}, n_{2}, n_{3}, \ldots, n_{k}, \ldots, n_{s}\right) \in C_{n_{k}}$. For each $j \in\left[n_{1}-n_{2}-1\right]$, the $\mathcal{D}$-edge $\left\{\left(n_{2}+\right.\right.$ $\left.j, 1, \ldots, 1),\left(n_{2}, n_{2}, n_{3}, \ldots, n_{k}, \ldots, n_{s}\right)\right\}$ implies that $\left(n_{2}+j, 1, \ldots, 1\right) \notin C_{n_{k}}$. From the $\mathcal{C}$ edge $\left\{(1,1, \ldots, 1),\left(n_{2}, n_{2}, n_{3}, \ldots, n_{k}, \ldots, n_{s}\right),\left(n_{2}, 1, \ldots, 1\right)\right\}$ and the $\mathcal{D}$-edge $\left\{\left(n_{k}, \ldots, n_{k}\right.\right.$, $\left.\left.n_{k+1}, \ldots, n_{s}\right),\left(n_{2}, 1, \ldots, 1\right)\right\}$, we get $\left(n_{2}, 1, \ldots, 1\right) \in C_{1}$. For $j \in\left[n_{1}-n_{2}-1\right]$, the $\mathcal{C}$-edge $\left\{\left(n_{2}+j, 1, \ldots, 1\right),\left(n_{2}, n_{2}, n_{3}, \ldots, n_{s}\right),\left(n_{2}, 1, \ldots, 1\right)\right\}$ implies that $\left(n_{2}+j, 1, \ldots, 1\right) \in C_{1}$.

For any $j \in\left[n_{1}-n_{2}\right]$, from the $\mathcal{D}$-edge $\left\{(1,1, \ldots, 1),\left(n_{2}+j, n_{2}, \ldots, n_{s}\right)\right\}$, we have $\left(n_{2}+j, n_{2}, \ldots, n_{s}\right) \notin C_{1}$. Moreover, the $\mathcal{C}$-edge $\left\{\left(n_{2}, n_{2}, n_{3}, \ldots, n_{s}\right),\left(n_{2}+j, 1, \ldots, 1\right),\left(n_{2}+\right.\right.$ $\left.\left.j, n_{2}, n_{3}, \ldots, n_{s}\right)\right\}$ implies that $\left(n_{2}+j, n_{2}, n_{3}, \ldots, n_{s}\right) \in C_{n_{k}}$ for any $j \in\left[n_{1}-n_{2}-\right.$ 1]. The fact that $\left\{\left(n_{1}, \ldots, n_{s}\right),\left(n_{2}, n_{2}, n_{3}, \ldots, n_{s}\right),\left(n_{2}, 1, \ldots, 1\right)\right\}$ is a $\mathcal{C}$-edge follows that $\left(n_{1}, n_{2}, n_{3}, \ldots, n_{s}\right) \in C_{n_{k}}$. Hence, $c=c_{k}^{s *}$. 
By the above discussion, the desired result follows.

Next, we shall construct another family of mixed hypergraphs.

Construction II. Let $X^{\prime \prime}=X_{n_{1}, \ldots, n_{s}}^{*} \backslash\left\{\left(n_{2}, 1, \ldots, 1\right)\right\}$ and $\mathcal{H}^{\prime \prime}=\mathcal{H}_{n_{1}, \ldots, n_{s}}^{*}\left[X^{\prime \prime}\right]$. Then, for any $i \in[s]$,

$$
c_{i}^{\prime \prime}=\left\{X_{i 1}^{\prime \prime}, X_{i 2}^{\prime \prime}, \ldots, X_{i n_{i}}^{\prime \prime}\right\}
$$

is a strict $n_{i}$-coloring of $\mathcal{H}^{\prime \prime}$, where $X_{i j}^{\prime \prime}=X^{\prime \prime} \cap X_{i j}^{s}, j \in\left[n_{i}\right]$.

Theorem 2.5 If $n_{1}=n_{2}+1$, the $\mathcal{H}^{\prime \prime}$ is a one-realization of $S$.

Proof. Referring to the proof of Theorem 2.4, all the strict colorings of $\mathcal{H}_{n_{2}, n_{2}, n_{3}, \ldots, n_{s}}^{*}$ are

$$
c_{i}^{\prime}=\left\{X_{i 1}^{\prime}, X_{i 2}^{\prime}, \ldots, X_{i n_{i}}^{\prime}\right\}, \quad i \in[s] \backslash\{1\},
$$

where $X^{\prime}=\left\{\left(x_{2}, x_{2}, x_{3}, \ldots, x_{s}\right) \mid x_{j} \in\left[n_{j}\right], j \in[s] \backslash\{1\}\right\}$ and $X_{i j}^{\prime}=X^{\prime} \cap X_{i j}^{*}, j \in\left[n_{i}\right]$.

For any strict coloring $c=\left\{C_{1}, C_{2}, \ldots, C_{m}\right\}$ of $\mathcal{H}^{\prime \prime}$, there are the following two possible cases.

Case 1. $\left.c\right|_{X^{\prime}}=c_{2}^{\prime}$. That is to say, $\left(i, i, x_{3}, \ldots, x_{s}\right) \in C_{i}$ under the coloring $c$ for any $\left(i, i, x_{3}, \ldots, x_{s}\right) \in X^{\prime \prime}$. By the proof of Theorem 2.4, $\left(n_{1}, n_{2}, n_{3}, \ldots, n_{s}\right) \notin C_{j}$ for any $j \in\left[n_{2}-1\right]$. Then, there are the following two possible subcases.

Case 1.1. $\left(n_{1}, n_{2}, n_{3}, \ldots, n_{s}\right) \in C_{n_{2}}$. It is immediate that $c=c_{2}^{\prime \prime}$.

Case 1.2 $\left(n_{1}, n_{2}, n_{3}, \ldots, n_{s}\right) \notin C_{n_{2}}$. Then $\left(n_{1}, n_{2}, n_{3}, \ldots, n_{s}\right) \in C_{n_{1}}$. It is immediate that $c=c_{1}^{\prime \prime}$.

Case 2. There exists a $k \in[s] \backslash\{1,2\}$ such that $\left.c\right|_{X^{\prime}}=c_{k}^{\prime}$. It is immediate that $\left(n_{k}, \ldots, n_{k}, n_{k+1}, \ldots, n_{s}\right) \in C_{n_{k}}$ and $(\underbrace{n_{k}, \ldots, n_{k}}_{k-1}, 1, \ldots, 1) \in C_{1}$. From the $\mathcal{C}$-edge $\left\{\left(n_{1}, n_{2}, \ldots, n_{s}\right),\left(n_{k}, \ldots, n_{k}, n_{k+1}, \ldots, n_{s}\right),\left(n_{k}, \ldots, n_{k}, 1, \ldots, 1\right)\right\}$ and the $\mathcal{D}$-edge $\left\{\left(n_{1}, n_{2}\right.\right.$, $\left.\left.\ldots, n_{s}\right),(1,1, \ldots, 1)\right\}$, we observe $\left(n_{1}, n_{2}, \ldots, n_{s}\right) \in C_{n_{k}}$. Therefore, $c=c_{k}^{\prime \prime}$.

Hence, the desired result follows.

Combining Lemma 2.2, Theorems 2.4 and 2.5, the proof of Theorem 1.1 is completed.

\section{Acknowledgment}

We wish to thank the referees for their helpful suggestions. The research is supported by NSF of Shandong Province (No. ZR2009AM013), NCET-08-0052, NSF of China (10871027) and the Fundamental Research Funds for the Central Universities of China. 


\section{References}

[1] C. Bujtás, Zs. Tuza, Uniform mixed hypergraphs: the possible numbers of colors, Graphs and Combin. 24 (2008), 1-12.

[2] T. Jiang, D. Mubayi, Zs. Tuza, V. Voloshin and D. West, The chromatic spectrum of mixed hypergraphs, Graphs and Combin. 18 (2002), 309-318.

[3] D. Kobler and A. Kündgen, Gaps in the chromatic spectrum of face-constrained plane graphs, Electronic J. Combin. 8 (2001), $\sharp$ N3.

[4] D. Král, On feasible sets of mixed hypergraphs, Electronic J. Combin. 11 (2004), $\sharp R 19$.

[5] A. Kündgen, E. Mendelsohn and V. Voloshin, Coloring of planar mixed hypergraphs, Electronic J. Combin. 7 (2000), $\sharp \mathrm{R} 60$.

[6] Zs. Tuza and V. Voloshin, Problems and results on colorings of mixed hypergraphs, Horizons of Combinatorics, Bolyai Society Mathematical Studies 17, Springer-Verlag, 2008, pp. 235-255.

[7] V. Voloshin, On the upper chromatic number of a hypergraph, Australasian J. Combin. 11 (1995), 25-45.

[8] V. Voloshin, Coloring Mixed Hypergraphs: Theory, Algorithms and Applications, AMS, Providence, 2002.

[9] V. Voloshin, Introduction to Graph and Hypergraphs Theory, Nova Scinece Publishers, Inc., New York, 2009.

[10] P. Zhao, K. Diao and K. Wang, The chromatic spectrum of 3-uniform bi-hypergraphs, Discrete Math. 311 (2011), 2650-2656. 